\title{
HYDROGEN PRODUCTION AND CARBON DIOXIDE RECOVERY FROM KRW OXYGEN-BLOWN GASIFICATION*
}

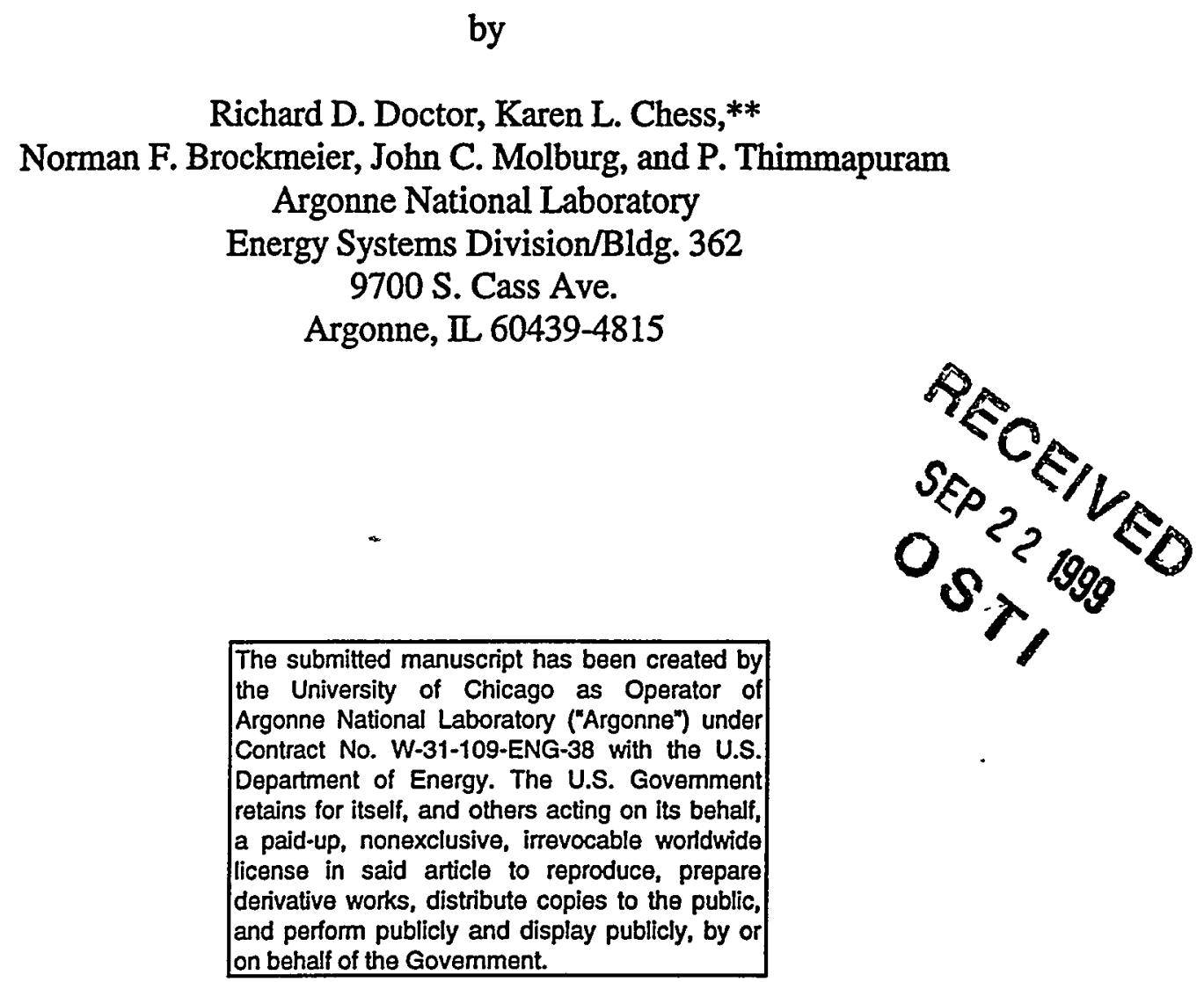

September 1998

Submitted for publication in the Proceedings of the Fourth Intl. Conference on Greenhouse Gas Control Technologies, Aug. 30-Sept. 2, 1998, Interlaken, Switizerland, sponsored by the IEA Greenhouse Gas R\&D Programme.

*Work supported by the U.S. Department of Energy, Office of Fossil Energy, under Contract No. W-31-109-ENG-38.

**Current address: Fuel Tech, 1001 Frontenac Rd., Naperville, II. 60563-1746 


\section{DISCLAIMER}

This report was prepared as an account of work sponsored . by an agency of the United States Government. Neither the United States Government nor any agency thereof, nor any of their employees, make any warranty, express or implied, or assumes any legal liability or responsibility for the accuracy, completeness, or usefulness of any information, apparatus, product, or process disclosed, or represents that its use would not infringe privately owned rights. Reference herein to any specific commercial product, process, or service by trade name, trademark, manufacturer, or otherwise does not necessarily constitute or imply its endorsement, recommendation, or favoring by the United States Government or any agency thereof. The views and opinions of authors expressed herein do not necessarily state or reflect those of the United States Government or any agency thereof. 


\section{DISCLAIMER}

Portions of this document may be illegible in electronic image products. Images are produced from the best available original document. 


\title{
HYDROGEN PRODUCTION AND CARBON DIOXIDE RECOVERY FROM KRW OXYGEN-BLOWN GASIFICATION
}

\author{
Richard D. Doctor, Karen L. Chess, ${ }^{*}$ Norman F. Brockmeier, John C. Molburg, and P. Thimmapuram \\ Argonne National Laboratory \\ 9700 S. Cass Ave. \\ Argonne, Il 60439-4815, USA
}

\begin{abstract}
An oxygen-blown KRW integrated gasification combined-cycle plant producing hydrogen, electricity, and supercritical$\mathrm{CO}_{2}$, was studied in a full-energy cycle analysis extending from the coal mine to the final destination of the gaseous product streams. A location in the mid-western United States was chosen $160-\mathrm{km}$ from Old Ben $\# 26$ mine which ships 3,866 tonnes/day of Illinois \#6 coal by diesel locomotive. Three parallel gasifier trains, each capable of providing $42 \%$ of the plant's $413.5 \mathrm{MW}$ nominal capacity use a combined total of 3,488 tonnes/day of $1 / 4$ " prepared coal. The plant produces a net $52 \mathrm{MW}$ of power and $3.71 \times 10^{6} \mathrm{~nm}^{3} /$ day of $99.999 \%$ purity hydrogen which is sent $100 \mathrm{~km}$ by pipeline at 34 bars. The plant also produces $3.18 \times 10^{6} \mathrm{~nm}^{3} /$ day of supercritical $\mathrm{CO}_{2}$ at 143 bars, which is sequestered in enhanced oil recovery operations $500 \mathrm{~km}$ away. $\mathrm{A} \mathrm{CO}_{2}$ emission rate of $1 \mathrm{kgCO} / \mathrm{kWh}$ was assumed for power purchases outside the fence of the IGCC plant.
\end{abstract}

\section{INTRODUCTION}

Oxygen-blown gasification is used to convert Illinois \#6 coal to synthesis gas [Fig. 1]. After particulate removal, a shift reactor uses steam to convert the $\mathrm{CO}$ component of the gas to $\mathrm{CO}_{2}$ and hydrogen $\left(\mathrm{H}_{2}\right)$. Next, $\mathrm{H}_{2} \mathrm{~S}$ is removed from the stream and processed to produce marketable sulfur. Carbon dioxide is then recovered in a glycol-based process and transported by pipeline for enhanced oil recovery. The gas stream after $\mathrm{CO}_{2}$ recovery is processed using pressure-swing adsorption (PSA) to recover $\mathrm{H}_{2}$ at a purity suitable for fuel cells, although there is no restriction on the actual hydrogen end-use. The $\mathrm{H}_{2}$ stream is transported to end users via pipeline, while the residual gas from PSA-a combination of hydrogen, methane, and light hydrocarbons-is used to generate electricity by combustion turbine combined cycle. Part of the electricity generated supplies the internal needs of the plant, and the excess is sent to the grid.

\section{MINING}

The assumed power plant location is $100 \mathrm{mi}(160 \mathrm{~km})$ by diesel-rail transport from the Old Ben \#26 underground mine in Sesser, Illinois. The plant receives 4,112 tons/day (155.4 metric tonnes/h) of $2 \times 4$-in. coal, which is prepared to $0 \times 1 / 4$ in. with $3.5 \%$ weight loss. A summary of this portion of the power cycle appears in Table 1.

\section{INTEGRATED GASIFICATION COMBINED CYCLE CONVERSION}

Previous process design studies to characterize integrated gasification combined-cycle (IGCC) power systems with $\mathrm{CO}_{2}$ capture technologies were modified using ASPEN $^{\circ}$ modeling to evaluate a configuration producing both merchant hydrogen and electricity $[1,2,3,4,5]$. The power plant configuration employs three parallel gasifier trains, each capable of providing $42 \%$ of the plantis $413.5 \mathrm{MW}$ nominal capacity (for the base case with no $\mathrm{CO}_{2}$ recovery.) After modification, the plant produces $131 \mathrm{MMscf} /$ day (3.71 million standard cubic m/day) of $99.999 \%$ purity hydrogen at $287.7 \mathrm{Btu} / \mathrm{scf}$; $119.9 \mathrm{KJ} / \mathrm{g}$ (LHV) which is sent $100 \mathrm{~km}$ by pipeline at 34 bars. At $100 \%$ efficiency, this could yield $460 \mathrm{MW}$ of power. The plant also produces $112 \mathrm{MMscf} /$ day ( 3.18 million standard cubic $\mathrm{m} /$ day) of supercritical- $\mathrm{CO}_{2}$ at 143 bars, which is sent $500-\mathrm{km}$ for sequestering in enhanced oil recovery. PSA reject gas goes to a turbine cycle to produce $118 \mathrm{MW}$. After supplying $66 \mathrm{MW}$ for internal power use this yields $52 \mathrm{MW}$ Net power. The designed plant availability is $95 \%$. This is largely reflected in higher projected maintenance costs.

\section{$\mathrm{H}_{2}$ PIPELINE}

A $100-\mathrm{km}$ pipeline design was prepared and costs were estimated for a high purity hydrogen flow of $3.71 \times 10^{6} \mathrm{~nm} / \mathrm{day}$ through a $343 \mathrm{~mm}$ pipe at 30 bar. There appears to be no economic justification for going to higher pipeline pressures and an internal study of the costs for delivering energy as methane vs. energy as $\mathrm{H}_{2}$ showed a $13 \%$ advantage for methane at 500 psi rising to a $46 \%$ advantage at 800 psi. Economic assumptions were for an availability of $95 \%$ and capital recovery of $12 \%$ to yield transmission costs of $0.171 \$ / \mathrm{Mscf} ; 0.564 \$ / \mathrm{GJ}$. It is very important to observe that the high costs of a dedicated pipeline dictate the high availabilities.

*Current address: Fuel Tech, 1001 Frontenac Rd., Naperville, II 60563-1746 


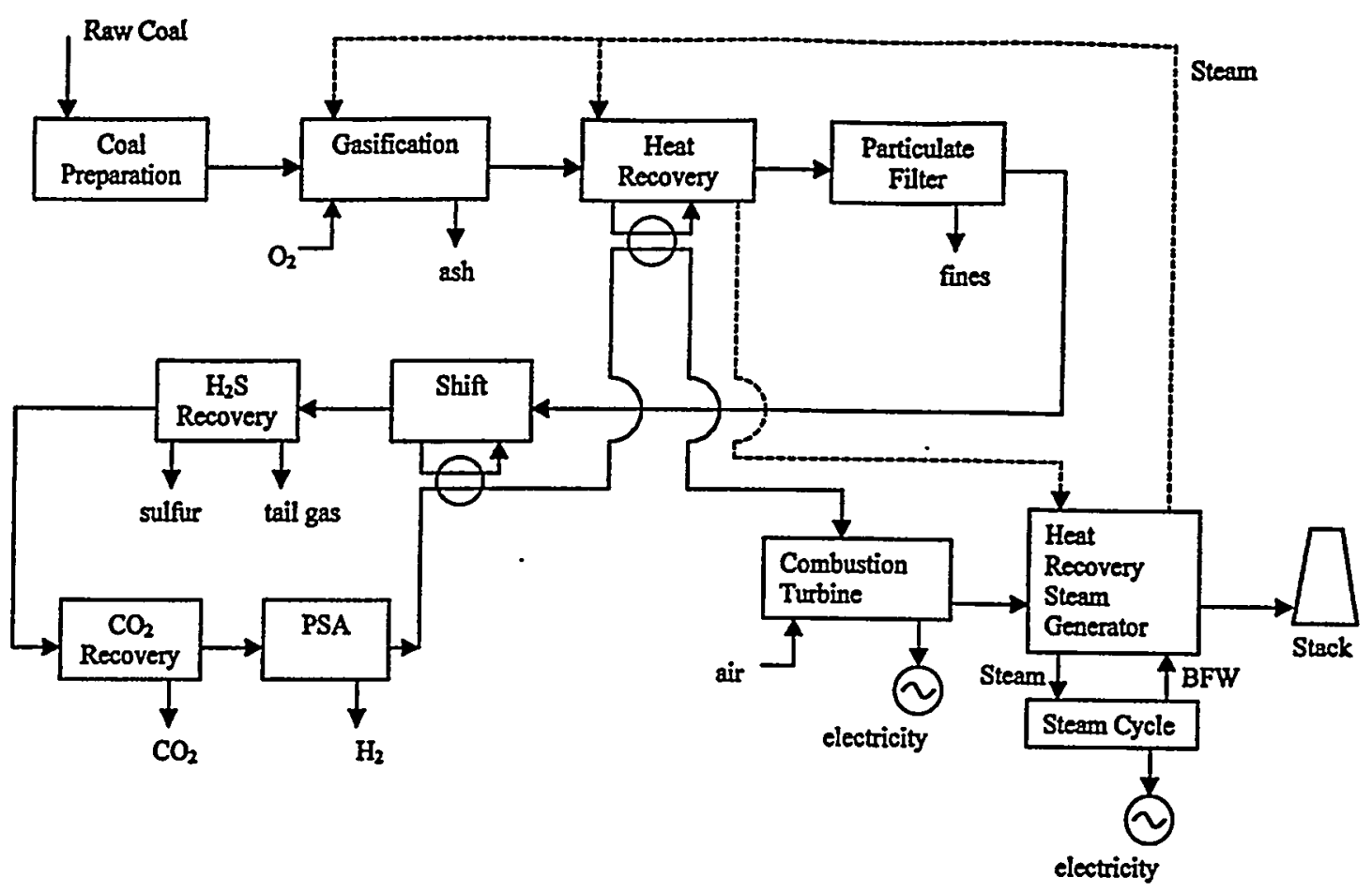

Fig. 1. Integrated Gasification Combined-Cycle Producing Electricity, $\mathrm{CO}_{2}$ and $\mathrm{H}_{2}$

Table 1. Energy Use in Coal Mining, Preparation, and Transportation

\begin{tabular}{|c|c|c|c|c|c|c|c|}
\hline metric units & $\begin{array}{l}\text { Electricity } \\
\mathrm{kWh} / \text { tonne }\end{array}$ & $\begin{array}{c}\text { Diesel } \\
\text { Fuel \#2 } \\
\text { tonne- } \\
\mathrm{km} / \mathrm{liter} \text {. }\end{array}$ & $\begin{array}{c}\mathrm{CO}_{2} \\
\text { Emissions } \\
\mathrm{kg} / \text { tonne } \\
\text { coal } \\
\end{array}$ & $\begin{array}{l}\text { Electricity } \\
\text { MW }\end{array}$ & $\begin{array}{l}\text { Losses } \\
\%\end{array}$ & $\begin{array}{l}\text { Coal } \\
\mathrm{kg} / \mathrm{h}\end{array}$ & $\begin{array}{l}\mathrm{CO}_{2} \\
\mathrm{~kg} / \mathrm{h}\end{array}$ \\
\hline \multicolumn{8}{|l|}{ MINING (a) } \\
\hline Methane emissions (b) & & & 9.63 & & $0.0 \%$ & 178,981 & 1,724 \\
\hline Hoisting & 6.12 & & 6.12 & & & & \\
\hline Drilling & 2.03 & & 2.03 & & & & \\
\hline Ventilation & 2.20 & & 2.20 & & & & \\
\hline Dewatering & 2.67 & & 2.67 & & & & \\
\hline Break and convey & 0.73 & & 0.73 & & & & \\
\hline \multirow[t]{2}{*}{ Ancillary } & 0.46 & & 0.46 & & & & \\
\hline & 14.21 & & 14.21 & 2.54 & $0.0 \%$ & 178,981 & 2,543 \\
\hline $\begin{array}{l}\text { PREPARATION } 2 \times 4 \text {-in. } \\
\text { TRANSPORT - } 161 \mathrm{~km}\end{array}$ & 0.44 & & 0.44 & 0.07 & $10.0 \%$ & 161,083 & 71 \\
\hline Mine to IGCC by rail & & 135 & 3.27 & & & & \\
\hline General service & 0.98 & & 0.98 & 0.15 & & & \\
\hline Return to mine & & 50 & 1.22 & & & & \\
\hline General service & 0.36 & & 0.36 & 0.06 & & & \\
\hline \multicolumn{2}{|l|}{ subtotal } & & 5.83 & 0.21 & $3.5 \%$ & 155,445 & 905 \\
\hline PREPARATION 1/4-in. (c) & 5.85 & & & & $6.5 \%$ & 145,341 & \\
\hline
\end{tabular}

(a) Operations of 250 days/yr at $13 \mathrm{hr} /$ day

(b) Methane emissions of $175 \mathrm{scf} /$ ton counted only as conversion to $\mathrm{CO}_{2}$ within a 14-yr life

(c) Accounted for in IGCC plant balance 


\section{$\mathrm{CO}_{2}$ PIPELINE}

Design and economic assumptions for a supercritical- $\mathrm{CO}_{2}$ pipeline were compared against current plans for Dakota Gasification Company, Beulah, ND [6] and Shell estimates of $\mathrm{CO}_{2}$ purchase costs at $\$ 3.25 / \mathrm{bbl}$ of oil recovered [7] with a reasonable $\mathrm{CO}_{2}$ utilization of $5.6 \mathrm{Mscf} / \mathrm{bbl}$ oil [8], which would come to a purchase price of about $\$ 0.60 / \mathrm{Mscf}$. Since, the 30-in. Shell Cortez line is unusually large - resulting in economies of scale - previously determined pipeline costs

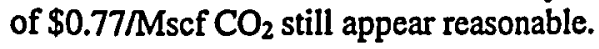

\section{RESULTS: FULL-ENERGY CYCLE BALANCES}

The energy costs of delivering electricity $100-\mathrm{km}$ from the IGCC plant are presented for three cases; the IGCC base case with no $\mathrm{CO}_{2}$ recovery (Table 2); the IGCC system with $\mathrm{CO}_{2}$ recovery (Table 3); the IGCC system developed for this study with $\mathrm{H}_{2}$ production and $\mathrm{CO}_{2}$ recovery (Table 4). For the Base-case with no $\mathrm{CO}_{2}$ recovery; delivered power was 396-MW full-cycle with emissions of $0.83 \mathrm{kgCO}_{2} / \mathrm{kWh}$. There is a derating with $\mathrm{CO}_{2}$ recovery. Delivered power becomes $366-\mathrm{MW}$ full-cycle at $0.20 \mathrm{kgCO} / \mathrm{kWh}$. An additional derating takes place in the present case with both $\mathrm{H}_{2}$ production and $\mathrm{CO}_{2}$ recovery where the hydrogen goes to 3-stage solid-oxide fuel cells. The delivered power now becomes $344-\mathrm{MW}$ full-cycle at $0.22 \mathrm{kgCO} / \mathrm{kWh}$. This is the combination of $52-\mathrm{MW}$ busbar at the plant and $298-\mathrm{MW}$ from fuel cells and a steam generator topping cycle.

Table 2. KRW $\mathrm{O}_{2}$-blown IGCC - Base Case Basis: Electric power delivery $100 \mathrm{~km}$ from station

\begin{tabular}{|c|c|c|c|c|c|c|c|}
\hline & $\mathrm{nm}^{3} / \mathrm{d}$ & tons/d & $\mathrm{kg} / \mathrm{h}$ & $\begin{array}{l}\text { Power } \\
\text { MW }\end{array}$ & $\begin{array}{l}\mathrm{CO}_{2} \\
\mathrm{~kg} / \mathrm{h}\end{array}$ & $\begin{array}{l}\mathrm{CH}_{4} \\
\mathrm{~kg} / \mathrm{h}\end{array}$ & $\begin{array}{l}\mathrm{N}_{2} \mathrm{O} \\
\mathrm{kg} / \mathrm{h}\end{array}$ \\
\hline \multicolumn{8}{|l|}{ MINING AND TRANSPORT } \\
\hline Coal methane emissions & & & & & & 566 & \\
\hline Mining operations \& preparation & & & & -2.61 & 2,614 & & 0.00003 \\
\hline Transport by rail - $161 \mathrm{~km}$ & & & & -0.21 & 905 & & 0.66265 \\
\hline $\begin{array}{l}\text { a. Subtotal } \\
\text { POWER PLANT }\end{array}$ & & & & -2.82 & 3,520 & 566 & 0.66267 \\
\hline Coal preparation (0-in. x 1/4-in.) & & 3,845 & 145,341 & -0.85 & & & \\
\hline $\mathrm{O}_{2}$ by cryogenic separation & $8,937,000$ & 2,347 & 88,717 & -29.29 & & & \\
\hline Steam from heat recovery generator & & & 17,254 & & & & \\
\hline Gasifier island & & & & -2.90 & - & & \\
\hline Solid waste & & 492 & 18,598 & & & & \\
\hline Sulfur & & 78 & 2,948 & -4.64 & & & \\
\hline $\mathrm{SO}_{2}$ (gasifier only) & & 6.92 & 262 & & 6,157 & & unknown \\
\hline Power island & . & & & -7.02 & 320,383 & & \\
\hline Miscellaneous (5\%) & & & & -2.24 & & & \\
\hline Subtotal & & & & -44.70 & 326,540 & & \\
\hline Power - gas turbine & & & & 627.40 & & & \\
\hline Power - air compressor and losses & & & & -328.60 & & & \\
\hline Power - steam turbine & & & & 159.40 & & & \\
\hline GROSS Power Subtotal & & & & 458.20 & & & \\
\hline b. NET Power & & & & 413.50 & & & \\
\hline $\begin{array}{l}\text { c. } \mathrm{CO}_{2} \text { PIPELINE AND } \\
\text { SEQUESTERING }\end{array}$ & & & & 0.00 & 0 & & \\
\hline d. $\mathbf{H}_{2}$ PIPELINE & & & & 0.00 & 0 & & \\
\hline $\begin{array}{l}\text { e. TRANSMISSION LOSS-3.5\% } \\
\text { f. NET ENERGY CYCLE -Base Case* }\end{array}$ & 0.833 & $\mathrm{~kg} \mathrm{CO}$ & $/ \mathbf{k W h}$ & $\begin{array}{l}-14.47 \\
396.20\end{array}$ & $\begin{array}{c}0 \\
330,060\end{array}$ & 566 & 0.66267 \\
\hline
\end{tabular}

$*_{\mathrm{f}}=\mathrm{a}+\mathrm{b}+\mathrm{c}+\mathrm{d}+\mathrm{e}$.

\section{APPLICATIONS}

Carbon dioxide as a supercritical product $(143 \mathrm{bar})$ can be recovered from coal gasification and power production. Where there is an enhanced oil recovery market, this actually is profitable. The need for high-pipeline utilization is critical. Hydrogen can be recovered at high purity (99.999\%) for sale from coal gasification, however the need for high pipeline-utilization is critical. Pressures of 35 bar are optimal. Fuel-cell conversion efficiencies need to approach 77\% to match the base-case output. At present, solid-oxide fuel cell efficiencies are 53-58\%; while alkaline fuel cell efficiencios are near $70 \%$. 
Table 3. $\mathrm{O}_{2}$-blown IGCC with $\mathrm{CO}_{2}$

Glycol $\mathrm{CO}_{2}$ and $\mathrm{H}_{2} \mathrm{~S}$ recovery; turbine topping

Basis: Electric power delivery $100 \mathrm{~km}$ from station

\begin{tabular}{|c|c|c|c|c|c|c|c|}
\hline & $\mathrm{nm}^{3} / \mathrm{d}$ & tons/d & $\mathrm{kg} / \mathrm{h}$ & $\begin{array}{l}\text { Power } \\
\text { MW }\end{array}$ & $\begin{array}{l}\mathrm{CO}_{2} \\
\mathrm{~kg} / \mathrm{h}\end{array}$ & $\begin{array}{l}\mathrm{CH}_{4} \\
\mathrm{~kg} / \mathrm{h}\end{array}$ & $\begin{array}{l}\mathrm{N}_{2} \mathrm{O} \\
\mathrm{kg} / \mathrm{h}\end{array}$ \\
\hline \multicolumn{8}{|l|}{ MINING AND TRANSPORT } \\
\hline Mining operations \& preparation & & & & -2.61 & 2,614 & & 0.00003 \\
\hline Transport by rail - $161 \mathrm{~km}$ & & & & -0.21 & 905 & & 0.66265 \\
\hline a. Subtotal & & & & -2.82 & 3,520 & 566 & 0.66267 \\
\hline \multicolumn{8}{|l|}{ POWER PLANT } \\
\hline Coal preparation (0-in. x 1/4-in.) & & 3,845 & 145,341 & -0.85 & & & \\
\hline $\mathrm{O}_{2}$ by cryogenic separation & $8,937,000$ & 2,347 & 88,717 & -29.29 & & & \\
\hline Steam from heat recovery generator & & & 17,254 & & & & \\
\hline Gasifier island & & & & -2.90 & & & \\
\hline Solid waste & & 492 & 18,598 & & & & \\
\hline Sulfur & & 78 & 2,948 & & & & \\
\hline $\mathrm{SO}_{2}$ (gasifier only) & & 6.92 & 262 & & 6,157 & & unknown \\
\hline Glycol circulation & & & & -5.80 & 320,383 & & \\
\hline Glycol refrigeration & & & & -4.50 & & & \\
\hline Power recovery turbines & & & & 3.40 & & & \\
\hline $\mathrm{CO}_{2}$ compression to pipeline (143 bar) & $3,178,000$ & & & -17.30 & $-260,055$ & & \\
\hline $\begin{array}{l}\text { Power island } \\
\text { Miscellaneous (5\%) }\end{array}$ & & & & $\begin{array}{l}-6.90 \\
-2.86\end{array}$ & & & \\
\hline Subtotal & & & & -67.01 & 66,485 & $\mathbf{0}$ & unknown \\
\hline Power - gas turbine & & & & 580.78 & & & \\
\hline Power - air compressor and losses & & & & -325.51 & & & \\
\hline Power - steam turbine & & & & 195.30 & & & \\
\hline GROSS Power Subtotal & & & & 450.57 & & & \\
\hline b. NET Power & & & & 383.56 & & & \\
\hline $\mathrm{CO}_{2}$ PIPELINE AND SEQUESTERING & $3,178,000$ & & & & 260,055 & & \\
\hline Pipeline booster stations & & & & -1.64 & 1,637 & & 0.00002 \\
\hline Geological reservoir (1\% loss) & & & & & $-257,454$ & & \\
\hline c. Subtotal & & & & -1.64 & 4,238 & 0 & 0.00002 \\
\hline d. $\mathrm{H}_{2}$ PIPELINE & & & & 0.00 & & & \\
\hline e. TRANSMISSION LOSS-3.5\% & & & & -13.42 & & & \\
\hline f. NET ENERGY CYCLE* & 0.203 & kg CO & $2 / \mathbf{k W h}$ & 365.67 & 74,242 & 566 & 0.66269 \\
\hline
\end{tabular}

$* f=a+b+c+d+e$. 
Table 4. KRW O2-blown IGCC

Glycol $\mathrm{CO}_{2}$ and $\mathrm{H}_{2} \mathrm{~S}$ recovery; $\mathrm{PSA} \mathrm{H}_{2}$ recovery; turbine topping; 3-stage solid oxide fuel cell

\begin{tabular}{|c|c|c|c|c|c|c|c|}
\hline & $\mathrm{nm}^{3} / \mathrm{d}$ & tons/d & $\mathrm{kg} / \mathrm{h}$ & $\begin{array}{l}\text { Power } \\
\text { MW }\end{array}$ & $\begin{array}{l}\mathrm{CO}_{2} \\
\mathrm{~kg} / \mathrm{h}\end{array}$ & $\begin{array}{l}\mathrm{CH}_{4} \\
\mathrm{~kg} / \mathrm{h}\end{array}$ & $\begin{array}{l}\mathrm{N}_{2} \mathrm{O} \\
\mathrm{kg} / \mathrm{h}\end{array}$ \\
\hline \multicolumn{8}{|l|}{ MINING AND TRANSPORT } \\
\hline Mining operations \& preparation & & & & -2.61 & 2,614 & 566 & 0.00003 \\
\hline Transport by rail - $161 \mathrm{~km}$ & & & & -0.21 & 905 & & 0.66265 \\
\hline a. Subtotal & & & & -2.82 & 3,520 & 566 & 0.66267 \\
\hline \multicolumn{8}{|l|}{ POWER PLANT } \\
\hline Coal preparation (0-in. x 1/4-in.) & & 3,845 & 145,341 & -0.85 & & & \\
\hline $\mathrm{O}_{2}$ by cryogenic separation & $8,937,000$ & 2,347 & 88,717 & -29.29 & & & \\
\hline Steam from heat recovery generator & & & 17,254 & & & & \\
\hline Gasifier island & & & & -2.90 & & & \\
\hline Solid waste & & 492 & 18,598 & & & & \\
\hline Sulfur & & 78 & 2,948 & & & & \\
\hline $\mathrm{SO}_{2}$ (gasifier only) & & 6.92 & 262 & & 6,157 & & unknown \\
\hline Glycol circulation & & & & -5.80 & 320,383 & & \\
\hline Glycol refrigeration & & & & -4.50 & & & \\
\hline Power recovery turbines & & & & 3.40 & & & \\
\hline $\mathrm{CO}_{2}$ compression to $143 \mathrm{bar}$ & $3,178,000$ & & & -17.30 & $-260,055$ & & \\
\hline $\mathrm{H}_{2}$ PSA purification to $31 \mathrm{bar}$ & $3,710,000$ & & & -3.18 & & & \\
\hline $\mathrm{H}_{2}$ cryo-storage for pipeline & & & & -0.92 & & & \\
\hline Power island & & & & -1.81 & & & \\
\hline Miscellaneous (5\%) & & & & -3.07 & & & \\
\hline Subtotal & & & & .66 .22 & 66,485 & 0 & unknown \\
\hline Power - gas turbine & & & & 244.53 & & & \\
\hline Power - air compressor and losses & & & & -169.48 & & & \\
\hline Power - steam turbine & & & & 42.93 & “ & & \\
\hline $\begin{array}{l}\text { GROSS Power Subtotal } \\
\text { b. NET Power }\end{array}$ & & & & $\begin{array}{c}117.98 \\
51.76\end{array}$ & & & \\
\hline the & & & & & & & \\
\hline $\begin{array}{l}\mathrm{CO}_{2} \text { PIPELINE \& } \\
\text { SEQUESTERING }\end{array}$ & $3,178,000$ & & & & 260,055 & & \\
\hline Pipeline booster stations & & & & -1.64 & 1,637 & & 0.00002 \\
\hline Geological reservoir ( $1 \%$ loss) & & & & & $-257,454$ & & \\
\hline c. Subtotal & & & & -1.64 & 4,238 & $\mathbf{0}$ & 0.00002 \\
\hline $\mathrm{H}_{2}$ PIPELINE OUTLET (21 bar) & $3,710,000$ & & & & & & \\
\hline $\mathrm{H}_{2}$ 3-stage SOFC ( $58 \%$ of $\left.460.0 \mathrm{MW}\right)$ & & & & 266.80 & & & \\
\hline Steam Generator ( $85 \%$ of $36.8 \mathrm{MW}$ ) & & & & 31.28 & & & \\
\hline d. Subtotal & & & & 298.08 & $\mathbf{0}$ & $\mathbf{0}$ & 0.00000 \\
\hline $\begin{array}{l}\text { e. TRANSMISSION LOSS-3.5\% } \\
\text { f. NET ENERGY CYCLE* }\end{array}$ & 0.216 & $\mathbf{k g ~ C}$ & $D_{2} / \mathbf{k W h}$ & $\begin{array}{l}-1.81 \\
343.56\end{array}$ & 74,242 & 566 & 0.66269 \\
\hline
\end{tabular}

\section{FULL ENERGY CYCLE ANALYSIS OF GREENHOUSE GAS FORCING}

Recent consideration of full-energy cycle analysis for power production (9) have emphasized the importance of greenhouse gases such as methane and $\mathrm{N}_{2} \mathrm{O}$ in addition to other than carbon dioxide. Modeling results suggest that a molecule of methane is equivalent to 56 molecules of $\mathrm{CO}_{2}$ in its climate-forcing impact, while each $\mathrm{N}_{2} \mathrm{O}$ molecule is equivalent to 280 molecules of carbon dioxide (10). These "equivalent $\mathrm{CO}_{2}$ impacts" were used as the basis for Fig. 2 which shows the equivalent $\mathrm{CO}_{2}$ emissions to provide $396-\mathrm{MW}$ of electricity $100-\mathrm{km}$ from the IGCC system. 


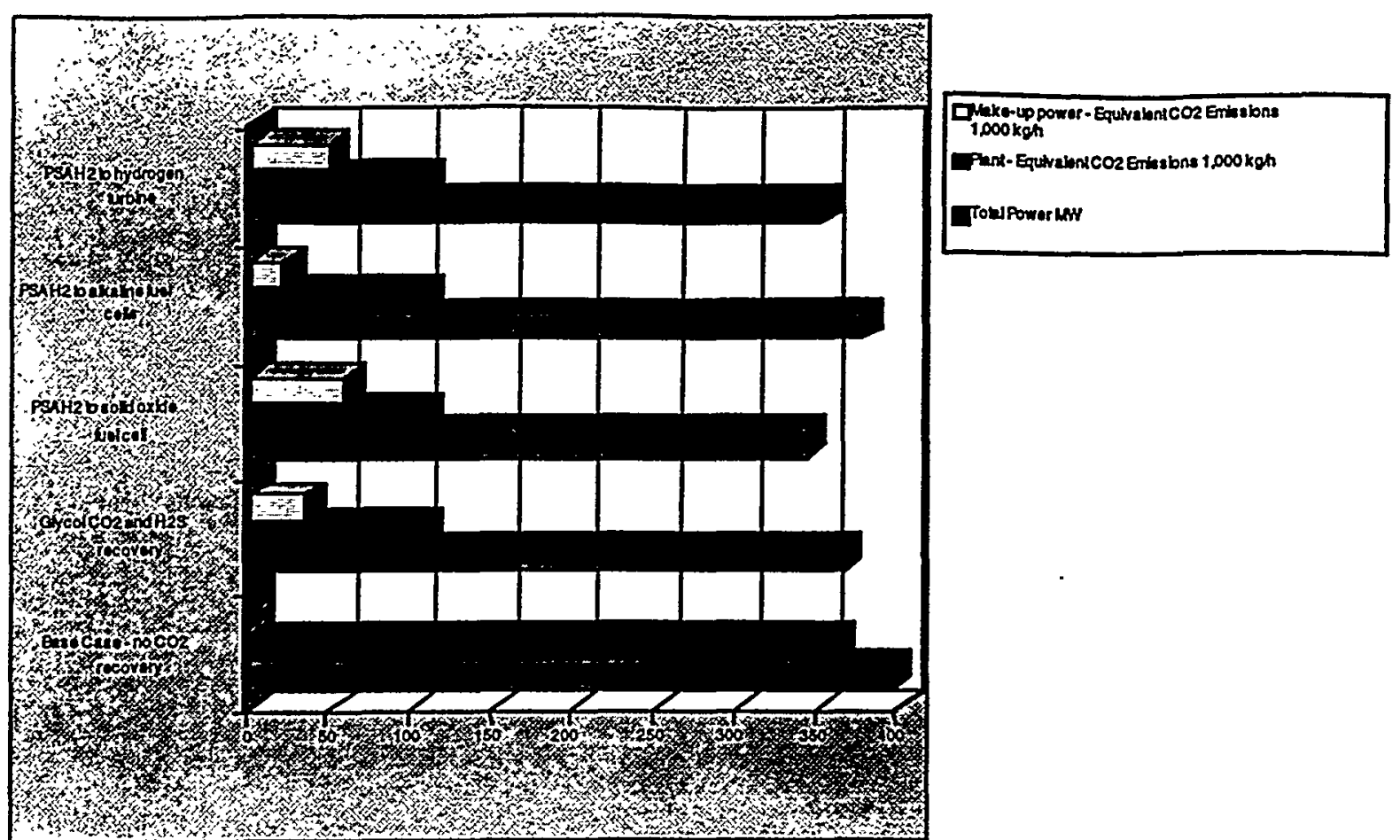

Fig. 2. Equivalent $\mathrm{CO}_{2}$ Greenhouse Emissions 396 MW Net-Cycle.

\section{ACKNOWLEDGMENTS}

This work is supported by the U.S. Department of Energy Fossil Energy Program at the Federal Energy Technology Center, through contract W-31-109-Eng-38; contract managers, Perry Bergman and Sean Plasynski. Special thanks for the support and encouragement from Harvey Ness and Dave Beecy.

\section{REFERENCES}

1. Gallaspy, D.T., et al., 1990. ISouthern Company Serviceís Study of a KRW-based GCC Power Plant,î EPRI GS-6876, Electric Power Research Institute, Palo Alto, CA.

2. Doctor, R.D., Molburg, J.C., Thimmapuram, P.R., Berry, G.F., and Livengood, C.D., 1994. IGasification Combined Cycle: Carbon Dioxide Recovery, Transport, and Disposal,î ANL/ESD-24, Argonne National Laboratory, Argonne, II.

3. Doctor, R.D., Molburg, J.C., and Thimmapuram, P.R., 1996. iKRW Oxygen-Blown Gasification Combined Cycle: Carbon Dioxide Recovery, Transport, and Disposal,î ANL/ESD-34, Argonne National Laboratory, Argonne, IL.

4. Doctor, R.D., J.C. Molburg, P.R. Thimmapuram, "Oxygen-Blown Gasification Combined Cycle, Carbon Dioxide Recovery, Transport, and Disposal," Proceedings of the 3rd Intl. Energy Agency Carbon Dioxide Disposal Symposium, Cambridge, MA, USA, 9-11 Sept. 1996, H.J. Herzog, Ed., Pergamon Press, Oxford; simultaneous publication in Energy Conservation and Management, 28 (Suppl.):575-580 (1997).

5. Doctor, R.D., Molburg, J.C., Thimmapuram, P.R., Berry, G.F., and Livengood, C.D., and Richard A. Johnson, IGasification Combined Cycle: Carbon Dioxide Recovery, Transport, and Disposal,î Proceedings of the 2nd Intl. Energy Agency Carbon Dioxide Disposal Symposium, Oxford, UK, 29-31 March, 1993, P.W. F. Reimer, ed., IEA Greenhouse Gas R\&D Programme, Pergamon Press, Oxford (1993); simultaneous Publication in Energy Conservation and Management, 34(9-11):1113-20 (1993).

6. iBig Canadian Miscible $\mathrm{CO}_{2}$ EOR Project," Oil \& Gas J. (July 7, 1997).

7. Moritis, C., iEOR Survey and Analysis,î Oil \& Gas J. (Apr. 15, 1996).

8. Hsu, C. et al., lProduction Report,î Oil and Gas J. (Oct. 23, 1995).

9. Smith, I.M., Greenhouse Gas Emission Factors for Coal-The Complete Fuel Cycle, International Energy Agency, London, UK, Nov. 1997.

10. Bryant, E., Climate Process and Change, Cambridge, 1997, p. 119. 